\title{
Hybrid delivery systems for methotrexate-lipidic liquid crystalline cubic phases and cubosomes with magnetic nanoparticles
}

\author{
Monika Mierzwa, Paweł Krysiński, Renata Bilewicz ${ }^{1}$ \\ Faculty of Chemistry, University of Warsaw, Pasteura 1, PL 02-093 Warsaw, Poland \\ ${ }^{1}$ Corresponding author: bilewicz@chem.uw.edu.pl
}

\begin{abstract}
The release profiles of methotrexate, an anticancer drug, from the monoolein liquid crystalline cubic phases were studied. The cubic phases were used either in the form of a lipidic film deposited onto a glassy carbon electrode surface or in the dispersed form of magnetocubosomes, which are considered a prospective hybrid drug delivery system. Commonly, cubosomes or liposomes are employed, but not in the case of toxic methotrexate, known to block receptors responsible for folate transport into the cells. The release profiles of the drug from the lipidic films were monitored electrochemically and described using the Higuchi model. They were also modified via changes in temperature; the release was faster, although deviating from the model when the temperature was increased. Magnetocubosomes - cubic phase nanoparticles containing hydrophobic magnetic nanoparticles placed in an alternating magnetic field of low frequency and amplitude, stimulated drug release from the suspension, which was monitored spectroscopically. These new biocompatible hybrid materials are very promising, allowing to control the release of the drug at the appropriate sites, but do require further investigations into their in vitro cytotoxicity and in vivo biodistribution.
\end{abstract}

Keywords: methotrexate, cubic phase, magnetocubosomes, monoolein, liquid crystalline phase, drug delivery system, alternating magnetic field

Abbreviations: MTX, methotrexate, SAXS, small angle x-ray scattering, LCP, liquid crystalline phase, DDS, drug delivery system, AMF, alternating magnetic field, MO,

\footnotetext{
${ }^{1}$ Corresponding author: Renata Bilewicz

E-mail address: bilewicz@ chem.uw.edu.pl

Faculty of Chemistry, University of Warsaw, Pasteura 1, 02-093, Warsaw, Poland

Tel: +48225526357
} 
monoolein, MNPs, magnetic nanoparticles, DLS, dynamic light scattering, Cryo-TEM, cryogenic transmission electron microscopy, DPV, differential pulse voltammetry, GC, glassy carbon, GCE glassy carbon electrode, SWV, square-wave voltammetry, CV, cyclic voltammetry, EE, entrapment efficiency

\section{Introduction}

Methotrexate (MTX) also known as amethopterin is an antimetabolite of folic acid. Its molecular structure differs from that of folic acid (FA) in two ways: first MTX has a 4-amino group instead of a hydroxyl group on the pteridine ring, and secondly it has a methyl group at the $\mathrm{N}^{10}$ position (Scheme 1).<smiles>[R14]Nc1nc(N)nc2ncc(CN(C)c3ccc(C(=O)NC(CCC(=O)O)C(=O)O)cc3)nc12</smiles><smiles>Nc1nc2ncc(CNc3ccc(C(=O)NC(CCC(=O)O)C(=O)O)cc3)nc2c(=O)[nH]1</smiles>

Scheme 1. Chemical structure of methotrexate (MTX) and folic acid (FA).

MTX blocks folate synthesis by inhibiting enzyme dihydrofolate reductase which synthesizes the active form of folate required for DNA and protein synthesis leading to major decrease in the cancer cell proliferation. [1] It is widely used for the treatment of acute leukemia, malignant lymphoma, osteogenic sarcoma, choriocarcinoma and other cancers (breast, head, neck, pulmonary and epidermoid). MTX is also used in the treatment of rheumatoid arthritis or other autoimmune diseases [2, 3] MTX is currently one of the most studied antitumor chemotherapeutic drugs since its therapeutic utility is limited by its high toxicity in the patient healthy cells where the levels of folates are also seriously reduced [4] The principal side-effects 
of MTX treatment are myelosuppression, thrombocytopenia, anemia, gastrointestinal mucositis, hepatitis, nausea, fatigue, headache and/or dyspnea. [3-6]

In view of the systemic side-effects of anticancer drugs, the design of drug carriers is very important. There are several methods for limiting MTX toxicity through its administration by means of carriers or targeting carriers, e.g., liposomes [7, 8], silica materials [9, 10], lipid nanoparticles [11, 12], chitosan nanoparticles [13], polymeric micelles with magnetic nanoparticles [14], nanorods [15], implants [16] and nanogels [17, 18].

A promising carrier is the liquid-crystalline lipidic cubic phase (LCP) that possesses a variety of desirable properties making them good candidates for drug delivery systems (DDS). LCP composed of monoolein (MO) is non-toxic, bioadhesive and biodegradable. The biodegradability of LCP is due to the fact that monoolein undergoes slow lipolysis in contact with esterase found in the tissues. The highly structured inverse bicontinuous LCP is composed of two identical, nonintersecting aqueous channels that are surrounded by curved lipid bilayers and can accommodate large amounts of active molecules of any polarity. [19] LCPs are stable in the presence of water, which is an important property in view of their perspective applications for DDS. The rate of drug release from liquid crystals can be modified by either changing the symmetry of the liquid crystal, the dimension of the water channel, or $\mathrm{pH}$ of the environment. Generally this can be achieved through manipulation of the lipid composition. [20-23] Previously, we studied the physical and chemical properties of hybrid materials composed of LCPs bearing hydrophilic and hydrophobic magnetic nanoparticles as a material for drug delivery and the outcome waspromising . [24] Magnetic nanoparticles and ions are currently of great interest in research related to medical applications [25] and are used as contrast agents for theranostics in medical imaging applications [26]. Few reported studies examined the effect of the addition of nanoparticles to liquid crystalline hexagonal phase [27], liposomes [28, 29] and cubosomes [30-32] creating a magnetic responsive system.

In this study, we focused on the delivery of MTX loaded monoolein hybrid systems. The MTX load and release studies were conducted for the monoolein cubic phase and cubosomes with and without hydrophobic magnetic nanoparticles present in the lipidic part of the carrier. Taking advantage of the presence of hydrophobic magnetic nanoparticles localized in the lipidic part of magnetocubosomes, we used an alternating magnetic field (AMF) with low frequency to stimulate the release of the drug from the carriers. 


\section{Experimental}

\section{Materials and Reagents}

\section{Materials}

All chemicals were of the highest quality and commercially available. Monoolein (1-oleoylrac-glycerol) (MO) (>99\% pure) was purchased from Hampton Research (Aliso Viejo, CA, USA) and used as received. Methotrexate (N-[4\{[2,4-diamino-6-pteridinyl-methyl]methylamino \}benzoyl] glutamic acid) (MTX) was obtained from Fluka ( $>98 \%$ pure) and used without further purification. Pluronic F-127 was purchased from Sigma-Aldrich. A $0.2 \mathrm{M}$ phosphate buffer was prepared by titrating $0.2 \mathrm{M}$ di-sodium hydrogen phosphate ( $>99 \%$ pure, ChemPur) with 0.2 M sodium di-hydrogen phosphate ( $>99 \%$ pure, Polish Chemicals Co.) to $\mathrm{pH}$ 7.4. All solutions were prepared using Milli Q water $\left(18.2 \mathrm{M} \Omega \cdot \mathrm{cm}^{-1}\right.$; Millipore, Bedford, MA, USA). To synthesize the hydrophobic nanoparticles, a $\mathrm{FeCl}_{3} \cdot 6 \mathrm{H}_{2} \mathrm{O}(\geq 98 \%$ pure, SigmaAldrich), $\mathrm{FeCl}_{2} \bullet 4 \mathrm{H}_{2} \mathrm{O}$ ( $\geq 99 \%$ pure, Sigma-Aldrich), $\mathrm{NH}_{4} \mathrm{OH}$ (25\% aqueous solution in $\mathrm{H}_{2} \mathrm{O}$, Chempur) and oleic acid (>99\% pure, Sigma-Aldrich) were used.

\section{Preparation of Cubic Phases and Cubosomes}

The LCPs were prepared by adding the appropriate amount of MTX solution to a $0.1 \mathrm{M}$ phosphate buffer in a glass vial with molten MO to obtain 59/1/40, 59.5/0.5/40 or $59.75 / 0.25 / 40 \%(w / w)$ for the phase MO/MTX/buffer.

Magnetic nanoparticles (MNPs) used to prepare hybrid cubic phases were synthesized as described in our previous report [24]. Briefly, we used the co-precipitation method with oleic acid as the capping agent. The synthesis was carried out under vigorous stirring and an argon atmosphere. As the first step, $\mathrm{FeCl}_{2} \bullet 4 \mathrm{H}_{2} \mathrm{O}(0.023 \mathrm{~mol})$ and $\mathrm{FeCl}_{3} \bullet 6 \mathrm{H}_{2} \mathrm{O}(0.046 \mathrm{~mol})$ were dissolved in $150 \mathrm{~mL}$ of deionized water. The mixture was heated to $45^{\circ} \mathrm{C}$ and then $11 \mathrm{~mL}$ of $25 \%$ ammonia was added, which caused appearance of black precipitate of nanoparticles. After half an hour, $3 \mathrm{~mL}$ of oleic acid was added. Then the suspension was heated to $80^{\circ} \mathrm{C}$ and kept at this temperature for one hour. Afterwards the $\mathrm{Fe}_{3} \mathrm{O}_{4}$ nanoparticles coated with oleic acid were washed several times with deionized water and ethanol under magnet-assisted sedimentation to remove the excess of oleic acid. After thorough washing, the nanoparticles of ca. $7 \pm 2 \mathrm{~nm}$ diameter were transferred to hexane as a stable suspension of controlled density. Their size distribution and mass contribution of organic shell (ca. 35\%) was discussed in our previous work $[24,29]$. Magnetic characterization of dry powder nanoparticles was 
carried out with QD vibrating sample magnetometer VSM, working at $300 \mathrm{~K}$ stabilized to $0.01 \mathrm{~K}$ accuracy, over the magnetic field range from 2.0 to $+2.0 \mathrm{~T}$. The saturation magnetization of as synthesized, oleic acid-coated nanoparticles was ca. $43 \mathrm{emu} / \mathrm{g}$, comparable with literature and our previous work [29].

To prepare the hybrid LCPs, the appropriate amount of MNPs dispersion in hexane was added to the molten MO and sonicated. The mixture was left in a desiccator to evaporate the solvent. Then, MTX, in phosphate buffer solution was added to the mixture. The ratio of the components of the hybrid system MO/NPs/MTX/buffer was 57.5/2/0.5/40\% (w/w). The samples were left in tightly sealed vials at room temperature in the dark until they became homogenous and transparent.

The procedure of preparing the magnetocubosome dispersion started with mixing molten MO and MNPs dispersion in hexane (34.5 mg of MNPs per $1 \mathrm{~g}$ of MO). After evaporation of the solvent, MTX was added (8.7 mg MTX per $1 \mathrm{~g}$ of MO). The solution was mixed followed by sonication until it became homogeneous. Then, the $1 \% \mathrm{~F}-127$ solution was added to obtain $94 \%$ $\mathrm{w} / \mathrm{w}$ and the mixture was dispersed by ultrasonication using a Sonics Vibra cell for $20 \mathrm{~min}(3 \mathrm{~s}$ “on”, 5 s “off”) at an amplitude of $40 \%$ of maximum output (130 watt, $30 \mathrm{kHz}$ ).

\section{Methods}

\section{Small-Angle X-ray Scattering}

The phase identity and structural parameters of the mesophases were determined using a SAXS instrument (Anton Paar), with a line collimation system and equipped with a sealed X-ray tube (PANalytical, $\lambda_{\mathrm{Cu}, \mathrm{K} \alpha}=0.1542 \mathrm{~nm}$ ) and a CCD detector (Princeton Instruments). The data was corrected for background scattering from the empty capillary and for slit-smearing effects by the desmearing procedure from the supplier's software using the Lake method.

The magnetocubosomes identity was also determined by SAXS with a GADDS system (Bruker) using a 3-pinhole collimation system working with $\mathrm{CuK \alpha}$ radiation $(\lambda \mathrm{Cu}, \mathrm{K} \alpha=0.1542$ $\mathrm{nm}$ ), a Nanostar system (Bruker) working with $\mathrm{CuK} \alpha$ radiation equipped with a Vantec 2000 area detector. The SAXS patterns were analyzed with the Bruker Topas 3 software.

Samples were placed into $2 \mathrm{~mm}$ glass capillaries, which were immediately sealed with the epoxy glue (UHU).

\section{Dynamic Light Scattering (DLS) and Zeta Potential}


The hydrodynamic diameter of the cubosomes was determined using a Malvern Zetasizer instrument (Nano ZS, UK) fitted with a $4 \mathrm{~mW} \mathrm{He}-\mathrm{Ne}$ laser $(\lambda=632.8 \mathrm{~nm})$ as the light source with a scattering angle of $173^{\circ}$. Samples were equilibrated for 2 min before measurement. The zeta potential was measured using the same instrument. The values were reported as averages from 3 measurements of each sample.

\section{Cryogenic Transmission Electron Microscopy (Cryo-TEM)}

A droplet of the cubosome sample was deposited on a lacey copper grid. The sample was then blotted with filter paper inside a climate chamber (Vitrobot Mark IV, FEI). The temperature was kept at $22{ }^{\circ} \mathrm{C}$ and $100 \%$ humidity. Afterwards, the grid was plunged into a liquid ethane bath cooled with liquid nitrogen. The cooled gird was maintained at a temperature of $-170{ }^{\circ} \mathrm{C}$ using a cryo holder 626 (Gatan). Specimens were observed with a TECNAI transmission electron microscope operating at $120-200 \mathrm{kV}$ acceleration voltage equipped with a $4 \mathrm{k} \mathrm{CCD}$ camera FEI Eagle.

\section{Electrochemical Measurements}

Electrochemical measurements were performed using a $\mathrm{CH}$ Instruments bipotentiostat model CHI750B. The three-electrode system consisted of a glassy carbon electrode (GC macroelectrode $\mathrm{A}=0.07 \mathrm{~cm}^{2}, \mathrm{GC}$ microelectrode $\left.\mathrm{A}=98.4 \mu \mathrm{m}^{2}\right), \mathrm{Ag}, \mathrm{AgCl} \mid 3 \mathrm{M} \mathrm{KCl}_{\text {aq }}$ reference electrode and Pt foil as a counter electrode. The GC electrodes were polished each time before measurement with alumina powder $(1.0,0.3$ and $0.05 \mu \mathrm{m})$ on a polishing cloth. The electrodes were rinsed with ultrapure water, sonicated in the ultrasonic bath and left to dry in air. Then, the working space of the GCE was covered with a ca. $1 \mathrm{~mm}$ thin film of cubic phase (which corresponds to ca. $5 \mathrm{mg}$ of the phase). The measurements were performed at $25^{\circ} \mathrm{C}$ and $37{ }^{\circ} \mathrm{C}$ using a BVT MT-1 minithermostat to maintain a constant temperature. All solutions were deoxygenated by passing high purity argon through the solution for $15 \mathrm{~min}$ before the measurement and the flow of pure gas over the solution was maintained during the experiment. The release profiles of MTX from LCPs were obtained by recording voltammetric peaks of MTX reduction using the differential pulse voltammetry technique (DPV). The experimental conditions were: pulse amplitude $50 \mathrm{mV}$, pulse width $70 \mathrm{mV}$, pulse period $0.2 \mathrm{~s}$. The saturation of the empty cubic phase with MTX was monitored using square wave voltammetry (SWV) with the following parameters: frequency $25 \mathrm{~Hz}$, potential increment $2 \mathrm{mV}$, and amplitude 0.01 $\mathrm{V}$. We also performed cyclic voltammetry $(\mathrm{CV})$ experiments on $\mathrm{GC}$ electrodes modified with 
a cubic phase. The $\mathrm{CV}$ experiments were carried out at various scan rates within the constant potential window of $-0.2 \mathrm{~V}$ (starting point) to $-1.2 \mathrm{~V}$. For each type of cubic phase, experiments were done in triplicate.

\section{Modeling of the Kinetics of Drug Release}

There are several literature models that describe the drug release from different matrices [33]. In accordance with our previous work [24], to determine the kinetics of the drug elution from the LCPs, the Korsmeyer-Peppas model was chosen. It describes the controlled drug release from polymeric systems similar to cubic phases. The kinetics of the drug release, according to this model, are described by the following equation:

$$
\frac{\mathrm{M}_{\mathrm{t}}}{\mathrm{M}_{\infty}}=\mathrm{kt}^{\mathrm{n}}
$$

where $\mathrm{M}_{\mathrm{t}} / \mathrm{M}_{\infty}$ is the fraction of drug released at time $\mathrm{t}$ versus $\mathrm{t}_{\infty}, k$ is the release rate constant and $n$ value gives details the release mechanism of the drug. To determine the value of $n$, the logarithm of less than initial $60 \%$ of the release data values were fitted versus the log of time. In the case of a cylindrical surface of a drug-containing carrier, when $n=0.45$, the diffusion mechanism corresponds to Fick's law; for $\mathrm{n}$ between 0.45 and 0.89 , the transport is non-Fickian, $\mathrm{n}=0.89$ corresponds to Case-II transport, and $\mathrm{n}>0.89$ for Super Case-II transport [33].

When $\mathrm{n} \approx 0.5$, the Korsmeyer-Peppas approach is simplified to the Higuchi model, which describes the drug release from semi-solid matrices. The Higuchi model is described by the following expression:

$$
\mathrm{M}_{\mathrm{t}} / \mathrm{M}_{\infty}=\mathrm{k}_{\mathrm{H}} \sqrt{\mathrm{t}}
$$

where $\mathrm{k}_{\mathrm{H}}$ is the Higuchi constant.

\section{Magnetic Field Generator}

The magnetic field generator was a custom-made inductor coil connected to an alternating electric power outlet $(20 \mathrm{~V}, 50 \mathrm{~Hz})$. The void of the inductor (stator) coil provided space for a quartz cuvette with a suspension of magnetocubosomes. Due to the design of the set-up, the magnetic field inside the inductor and quartz cuvette was not isotropic and its maximum value was measured as $10 \mathrm{mT}$. A constant temperature was maintained $\left(22^{\circ} \mathrm{C}\right)$ during the release experiments and the coil was cooled using a fan [29]. 


\section{Spectroscopic Measurements}

AMF-stimulated drug release. The release experiments from magnetocubosomes were performed using a UV-Vis Cary 60 spectrophotometer (Agilent Technologies) with a $1 \mathrm{~cm}$ quartz cuvette in the wavelength range from 600 to $250 \mathrm{~nm}$. A series of samples, each containing $0.5 \mathrm{mg} / \mathrm{mL}$ magnetocubosome dispersion in $0.1 \mathrm{M}$ phosphate buffer at $\mathrm{pH} 7.4$ was prepared. Each of the samples was subsequently placed in the cuvette inside the inductor coil for a specified time. Then, the cuvette was removed from the inductor coil and the absorbance was measured at $303 \mathrm{~nm}$. For each experiment a fresh sample was used.

The spectroscopic measurements were also used to calculate the entrapment efficiency $(\%)$ of MTX in magnetocubosomes. The freshly prepared magnetocubosomes were diluted ten times. Then, the diluted sample was placed in an Amicon Ultra- 0.5 centrifugal filter unit with an Ultracel-3 membrane (NMWL $3 \mathrm{kDa}$, Sigma Aldrich, Germany) and centrifuged for $20 \mathrm{~min}$ at $6000 \mathrm{rpm}$ to separate the unbound drug. The separated aqueous phase with free drug was ten times diluted in $0.1 \mathrm{M}$ phosphate buffer and the absorbance was measured at $303 \mathrm{~nm}$. The entrapment efficiency (EE) was determined using the following expression:

$$
E E=\frac{C_{T}-C_{F}}{C_{T}} \cdot 100 \%
$$

where $C_{\mathrm{T}}$ is the concentration of the drug added to the cubosome during preparation and $C_{\mathrm{F}}$ is the concentration of the free drug in the ultrafiltrate detected after centrifugation.

The standard calibration curve for MTX solution in $0.1 \mathrm{M}$ phosphate buffer at $\mathrm{pH} 7.4$ was developed from at least nine dilutions in the concentration range of $20.0-0.89 \mu \mathrm{g} / \mathrm{mL}$. The absorption spectra of MTX buffer solutions were measured in the wavelength range of 600-250 $\mathrm{nm}$ with the characteristic $\lambda$ at $303 \mathrm{~nm}$. All measurements were performed at $25^{\circ} \mathrm{C}$.

\section{Results and Discussion}

\section{Structural Characterization of the MTX Doped Cubic Phases}

It is well known that a monoolein/water mixture in a ratio of $60 / 40 \% \mathrm{w} / \mathrm{w}$ forms a bicontinuous cubic phase characterized by $P n 3 m$ symmetry [34 - 36]. The presence of MTX up to $2 \% \mathrm{w} / \mathrm{w}$ did not compromise the internal structure of the monoolein bicontinuous cubic phase. The size 
of the drug is smaller than the length of the unit cell of the cubic phase. The SAXS data of the pure MO phase and those doped with $2 \%$ and $1 \% \mathrm{w} / \mathrm{w}$ of MTX at $25^{\circ} \mathrm{C}$ and $37{ }^{\circ} \mathrm{C}$ are shown in Fig. 1. The peak positions of a cubic lattice are observed at both temperatures. These peak positions are consistent with the cubic phase of the $P n \overline{3 m}$ space group (characteristic Bragg diffractions: $\sqrt{2}: \sqrt{ } 3: \sqrt{ } 4 \sqrt{6}: \sqrt{ } 8: \sqrt{ } 9$ are shown near the corresponding peaks in Fig. 1). The calculated values of the lattice parameter, lipid length and diameter of the water channels are listed in Table 1. The values were calculated from the SAXS spectra (for more details see S1 and Kulkarni et al. [19]) Incorporation of MTX into the monoolein phase did not affect the lattice parameter or the size of the water channels (Table 1), and noticeable differences of ca. 1 $\mathrm{nm}$ are on the verge of error. 


\begin{tabular}{|c|c|c|c|c|c|}
\hline MO/aq* & 25 & Pn3 m & 10.2 & 1.7 & 4.5 \\
\hline $60 / 40 \%$ & 37 & Pn3 m & 9.5 & 1.6 & 4.2 \\
\hline \multirow{2}{*}{$\begin{array}{c}\text { MO/MTX/aq* } \\
59 / 1 / 40 \%\end{array}$} & 25 & Pn3 m & 10.3 & 1.8 & 4.5 \\
\hline & 37 & Pn3 m & 9.8 & 1.7 & 4.3 \\
\hline \multirow{2}{*}{$\begin{array}{c}\text { MO/MTX/aq* } \\
58 / 2 / 40 \%\end{array}$} & 25 & Pn3 m & 10.3 & 1.8 & 4.6 \\
\hline & 37 & Pn3 m & 9.7 & 1.6 & 4.3 \\
\hline
\end{tabular}

*'aq' is used as an abbreviation for the phosphate buffer

\section{Electrochemical Measurements}

\section{Methotrexate Incorporated in the Monoolein Cubic Phase}

MTX is an electroactive molecule that undergoes two redox processes. 1) The oxidation of MTX pyrazine moiety to 7-hydroxymethotrexate, which is an irreversible process occurring at positive potentials with the exchange of $2 \mathrm{e}^{-} / 2 \mathrm{H}^{+}$. 2) The reduction at negative potentials of the diamino-pteridinyl moiety to its 5,8 -dihydro derivative via transfer of $2 \mathrm{e}^{-} / 2 \mathrm{H}^{+}$, which is also reversible (Scheme S2). [37]

The electrochemical behavior of MTX was investigated using cyclic (CV), square wave (SWV) and differential pulse voltammetry (DPV) in $0.2 \mathrm{M}$ phosphate buffer, $\mathrm{pH}$ 7.4. Previous reports have shown that the electrochemical response of MTX to CGE differs depending on buffer $\mathrm{pH}$. $[37,38]$ Even though the obtained peaks were well resolved in acidic media, our main goal was to examine the drug's behavior at physiological $\mathrm{pH}$. We began our investigations by examining the redox processes of MTX at an electrode covered with LCP saturated with the drug. This was achieved by dipping the electrode modified with monoolein LCP in $2.33 \mathrm{mM}$ solution of MTX in $0.2 \mathrm{M}$ phosphate buffer at room temperature. The process of cubic phase saturation with MTX was monitored using the highly sensitive SWV method. After 15 min, the peak correlating to drug reduction was observed. Saturation was reached after 8 h (Fig. 2). When a constant SWV peak current was attained, cyclic voltammograms were recorded at different scan rates. The dependencies of the reduction peak currents on the square root of the scan rate was linear $\left(\mathrm{R}^{2}=0.990\right)$, proving the diffusion control of the electrode processes (Fig. 2 inset). 


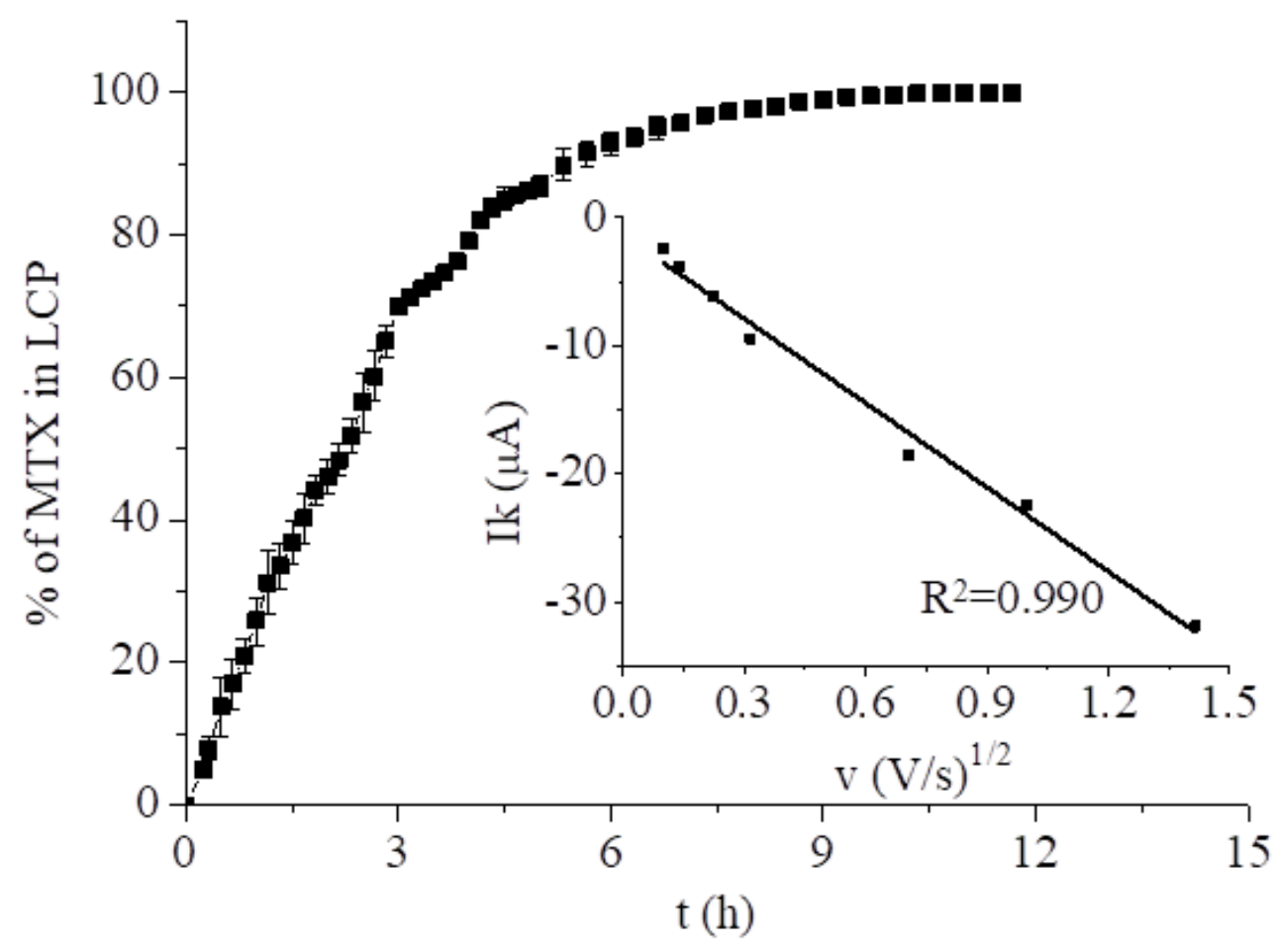

Fig. 2. Saturation of LCP with drug from $2.33 \mathrm{mM}$ MTX solution in $0.2 \mathrm{M}$ phosphate buffer monitored by SWV. Inset: Dependence of the CV reduction peak current at $-0.790 \mathrm{~V}$ vs. the square root of the scan rate for the cubic phase layer phase with MTX after $12 \mathrm{~h}$ of saturation.

In the next step, MTX was incorporated into the monoolein cubic phase at several concentrations: $0.10,0.25,0.50$ and $1.00 \% \mathrm{w} / \mathrm{w}$. Drug loaded cubic phases were used to modify the normal size GCE and GC microelectrode. The working space of the electrodes was modified with a thin film of cubic phase (ca. $1 \mathrm{~mm}$ ) containing MTX and transferred to the cell filled with deoxygenated phosphate buffer at room temperature. The response of the drug in negative potentials was monitored using DPV for the normal sized electrode and CV for the microelectrode. In the case of GCE, the transport to the electrode surface was determined by linear diffusion and recorded as a peak. The DPV reduction peak currents of the drug appeared ca. $-0.790 \mathrm{~V}$ and increased with MTX concentration (Fig. 3A). In the case of the microelectrode, the diffusion of the electroactive molecules was spherical and the CV response was in the form of a wave (Fig. 3B). The wave height increased with the amount of MTX in the LCP. In both cases, the dependence of the MTX response on the drug concentration in the LCP was linear (Fig. 3 inset). 

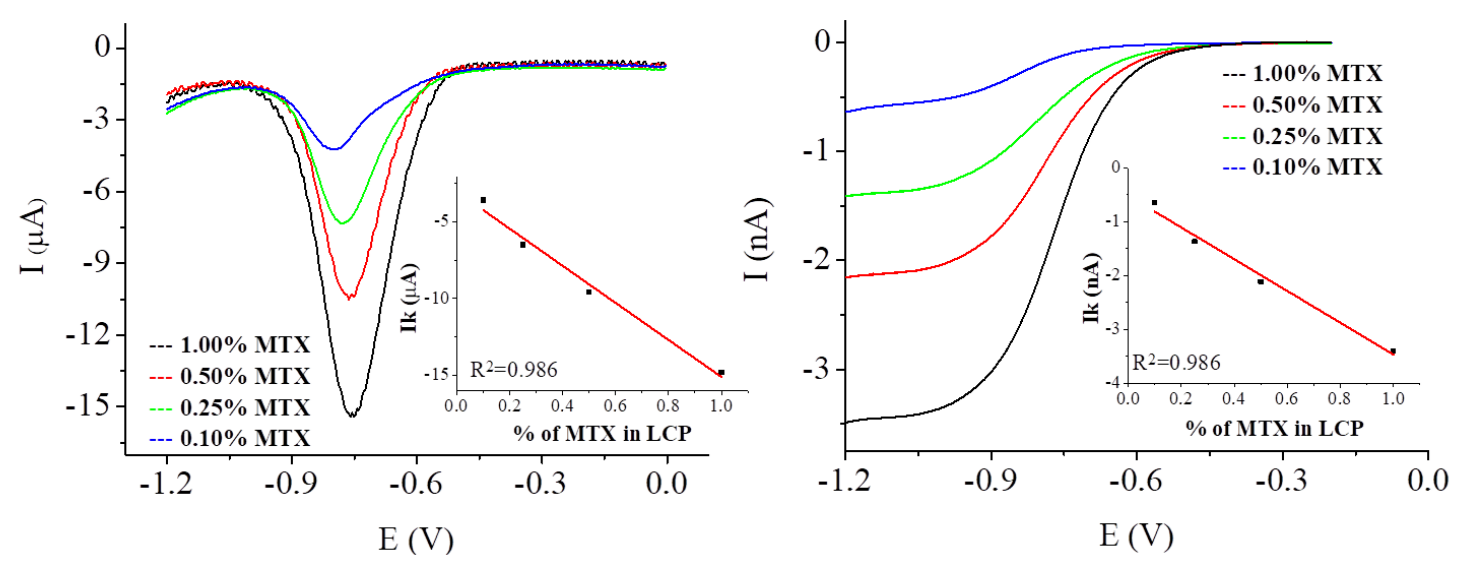

Fig. 3. DPV recorded from a GCE (A) and CV from a GC microelectrode (B) modified with the cubic phase containing 1.00, 0.50, 0.25 and $0.10 \% \mathrm{w} / \mathrm{w}$ of MTX at $\mathrm{pH} 7.4$. Inset: Dependence of the MTX response on the drug concentration in the LCP.

Drug elution studies of MTX from monoolein cubic phases were conducted at several concentrations: $0.25,0.50$ and $1.00 \% \mathrm{w} / \mathrm{w}$. The measurements were performed under an argon atmosphere at $25^{\circ} \mathrm{C}$ and $37^{\circ} \mathrm{C}$. The drug release profiles were investigated based on the changes in the DPV voltammograms with time counting from the moment of immersion of the electrodes in the pristine buffer. Over the course of time, the peak current decreased, which directly shows the elution of the drug. The dependence of the peak current on measurement time is shown in Fig. S3. The results were plotted as the normalized current (I/Io) vs. time and are presented in Fig. 4. Fig. 4A demonstrates the elution of the drug at room temperature. The profiles of the drug release were similarly independent of the initial concentration of the drug in the cubic phase. In all cases, the total elution was reached after ca. $350 \mathrm{~min}$, and 50\% w/w $\operatorname{MTX}\left(\mathrm{T}_{50}\right)$ was released after $42 \mathrm{~min}$ for 0.25 and $0.50 \% \mathrm{w} / \mathrm{w}$ MTX and after $40 \mathrm{~min}$ for $1.00 \%$ w/w MTX. Fig. 4B shows the elution of MTX at $37{ }^{\circ} \mathrm{C}$, with the total elution of the drug being achieved after $250 \mathrm{~min}$. $\mathrm{T}_{50}$ occurred after ca. $32 \mathrm{~min}$ for 0.25 and $0.50 \%$ MTX and $29 \mathrm{~min}$ for $1.00 \%$ MTX. These results are attributed to the faster moving molecules at higher temperatures. 

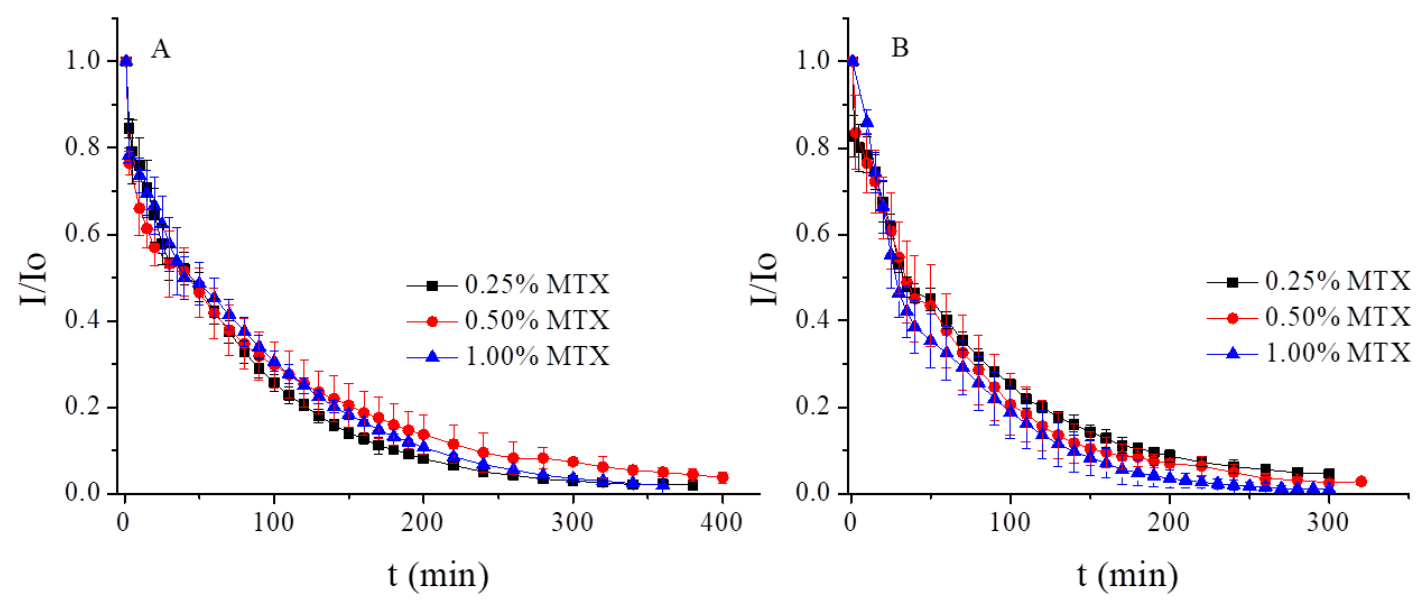

Fig. 4. Release profile of MTX from LCPs at $\mathrm{pH} 7.4$ at $25^{\circ} \mathrm{C}(\mathrm{A})$ and $37^{\circ} \mathrm{C}(\mathrm{B})$.

All drug release data were plotted as a log percentage of the drug release vs log time. The slope of the plot allowed evaluation of the exponent $n$ in the Korsmeyer-Peppas model. The results obtained for MTX at $25{ }^{\circ} \mathrm{C}$ gave an $n$ value close to 0.45 , which means that the diffusion mechanism corresponds to Fick's laws and the release mechanism proceeds according to the Higuchi model. With increasing temperature, the $n$ value in all cases starts to deviate from 0.45 , indicating the influence of the LCP matrix and a transition to non-Fickian transport (see Table 2).

Table 2. Release characteristics of methotrexate from LCPs.

\begin{tabular}{|c|c|c|c|c|c|}
\hline & \multicolumn{3}{|c|}{ Korsmeyer-Peppas } & \multicolumn{2}{c|}{ Higuchi } \\
\hline \% MTX & $\mathrm{N}$ & $\mathrm{R}^{2}$ & $\mathrm{k}\left[\% / \mathrm{h}^{\mathrm{n}}\right]$ & $\mathrm{k}_{\mathrm{H}}[\% / \mathrm{h}]$ & $\mathrm{R}^{2}$ \\
\hline $\mathbf{1 . 0}$ at $\mathbf{2 5}{ }^{\circ} \mathbf{C}$ & $0.44 \pm 0.05$ & $0.986 \pm 0.004$ & $56.08 \pm 5.40$ & $47.49 \pm 1.49$ & $0.977 \pm 0.010$ \\
\hline $\mathbf{1 . 0}$ at $37^{\circ} \mathbf{C}$ & $0.65 \pm 0.19$ & $0.921 \pm 0.102$ & $66.36 \pm 6.32$ & $\mathrm{a}$ & $\mathrm{a}$ \\
\hline $\mathbf{0 . 5}$ at $25^{\circ} \mathbf{C}$ & $0.41 \pm 0.03$ & $0.967 \pm 0.006$ & $62.34 \pm 2.22$ & $40.51 \pm 9.49$ & $0.943 \pm 0.038$ \\
\hline $\mathbf{0 . 5}$ at $\mathbf{3 7}$ & & & & & \\
${ }^{\circ} \mathbf{C}$ & $0.56 \pm 0.11$ & $0.950 \pm 0.043$ & $61.38 \pm 8.76$ & $66.22 \pm 4.21$ & $0.966 \pm 0.020$ \\
\hline
\end{tabular}




\begin{tabular}{|c|c|c|c|c|c|}
\hline $\begin{array}{c}\mathbf{0 . 5} \text { and } \\
\mathbf{2 \%} \text { MNPs } \\
\text { at } 25{ }^{\circ} \mathbf{C}\end{array}$ & $0.47 \pm 0.06$ & $0.949 \pm 0.014$ & $60.60 \pm 2.73$ & $62.68 \pm 5.85$ & $0.945 \pm 0.021$ \\
\hline $\begin{array}{c}\mathbf{0 . 2 5} \text { at } 25 \\
{ }^{\circ} \mathbf{C}\end{array}$ & $0.45 \pm 0.08$ & $0.975 \pm 0.005$ & $57.12 \pm 3.80$ & $53.97 \pm 3.38$ & $0.982 \pm 0.008$ \\
\hline $\begin{array}{c}\mathbf{0 . 2 5} \text { at 37 } \\
{ }^{\circ} \mathbf{C}\end{array}$ & $0.57 \pm 0.09$ & $0.943 \pm 0.014$ & $63.51 \pm 4.18$ & $72.21 \pm 4.11$ & $0.962 \pm 0.009$ \\
\hline
\end{tabular}

a: not determined

\section{Behavior of MTX Incorporated into Hybrid LCP Systems}

We have previously reported that incorporation of up to $2 \% \mathrm{w} / \mathrm{w}$ of hydrophobic magnetic nanoparticles (MNPs) has no effect on the phase symmetry [24] Due to its size (ca. $7 \mathrm{~nm}$ ) and lipid coating, MNPs are located partly in the lipidic domain of LCP and the water channels are not occupied. The magnetic nanoparticles do not block access of the drug to the electrode surface. A hybrid phase with MTX led to a similar outcome. MTX release profiles from the monoolein hybrid cubic phase containing $2 \% \mathrm{w} / \mathrm{w}$ of MNPs and $0.5 \% \mathrm{w} / \mathrm{w}$ of drug were monitored using the DPV method (Fig. 5, S4). The measurements were carried out at $25^{\circ} \mathrm{C}$ under an argon atmosphere. Incorporation of $2 \% \mathrm{w} / \mathrm{w}$ MNPs resulted in an increase of the initial peak current of MTX by $4 \mu \mathrm{A}$, without a change in the peak potential, $-0.790 \mathrm{~V}$ (Fig. S4). In the presence of MNPs, the mechanism of the drug release at room temperature is similar the MO phase. The total elution of MTX is maintained at the same level as pure monoolein phase. In this case, the value of $\mathrm{T}_{50}$ is $35 \mathrm{~min}$ and the $n$ value of ca. 0.45 suggests that the drug is released according to Fick's law (see Table 2). 


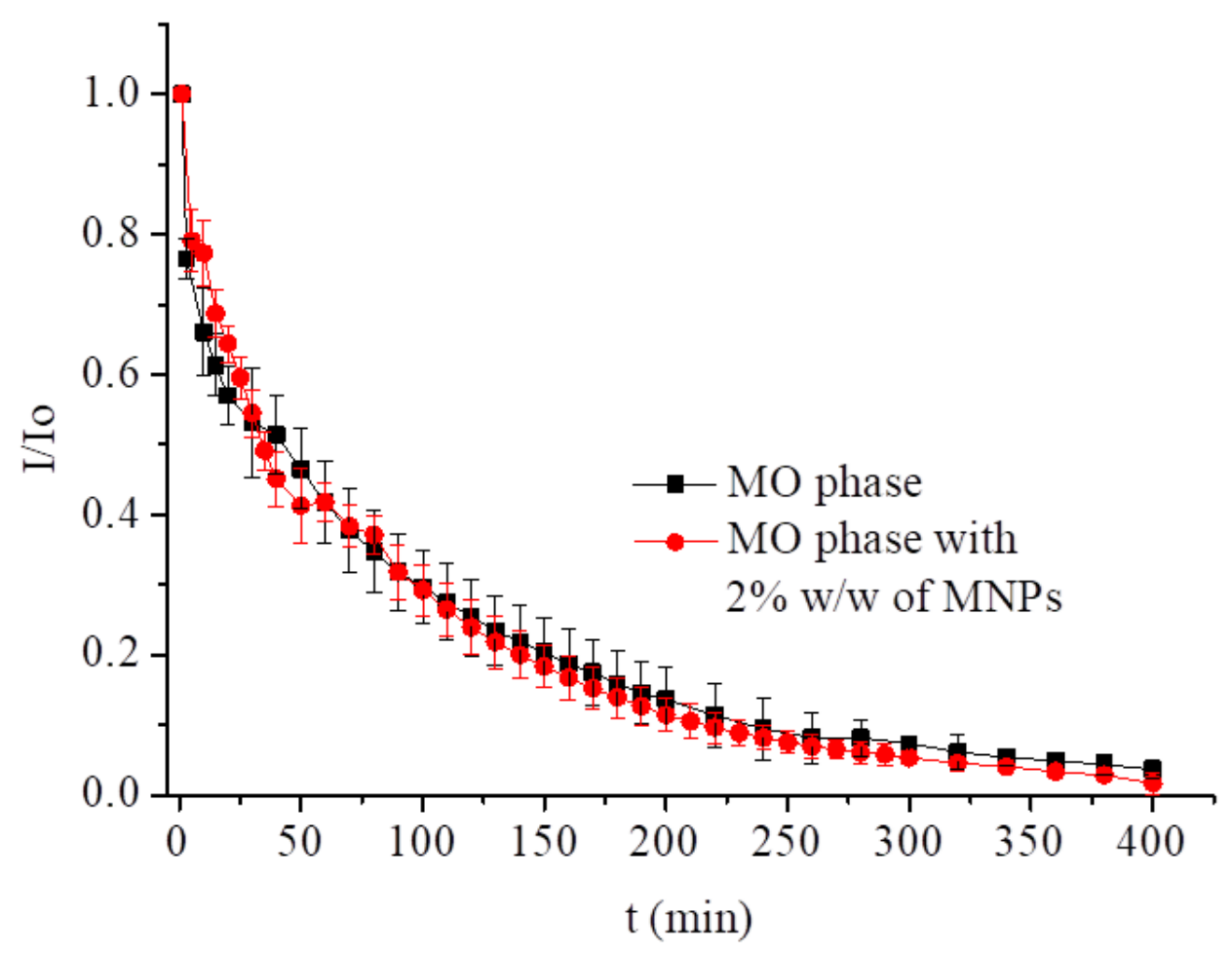

Fig. 5. Release profiles of MTX from LCPs with and without magnetic nanoparticles at $\mathrm{pH} 7.4$ at $25^{\circ} \mathrm{C}$.

\section{MTX Incorporated into Magnetocubosomes}

Nanoparticles of the hybrid cubic phase, magnetocubosomes, can be used for magnetic drug targeting, hyperthermia treatment, or magnetic resonance imaging [39] due to their small size and magnetic properties that allow them to move in a magnetic field. When loaded with the appropriate anti-cancer drug, such as MTX, they can serve as a platform for drug targeting delivery systems. Cubosomes and magnetocubosomes loaded with MTX were prepared, and their characteristics evaluated. The cubic nature of the LCP nanoparticles was characterized by SAXS. The SAXS pattern for cubosomes with MTX shows the relative positions of the diffraction signals $-\sqrt{2}: \sqrt{ } 4: \sqrt{ } 6$ - corresponding to the $\operatorname{Im} \overline{3 m}$ space group (Fig. 6 , black line). In the case of magnetocubosomes identical signals are observed (Fig. 6, red line). The crystallographic lattice parameter is $13.9 \mathrm{~nm}$ and $14.3 \mathrm{~nm}$ for cubosomes and magnetocubosomes, respectively. The diameter of magnetocubosomes doped with MTX was evaluated using DLS measurements, which was ca. $130 \mathrm{~nm}$, and the zeta potential was ca. -30 $\mathrm{mV}$ (Fig. S5). The entrapment efficiency of MTX in the magnetocubosomes was evaluated 
using equation 3 and a standard calibration curve for MTX solution in $0.1 \mathrm{M}$ phosphate buffer at pH 7.4 (Fig. S6). The load efficiency was $63.1 \%$.

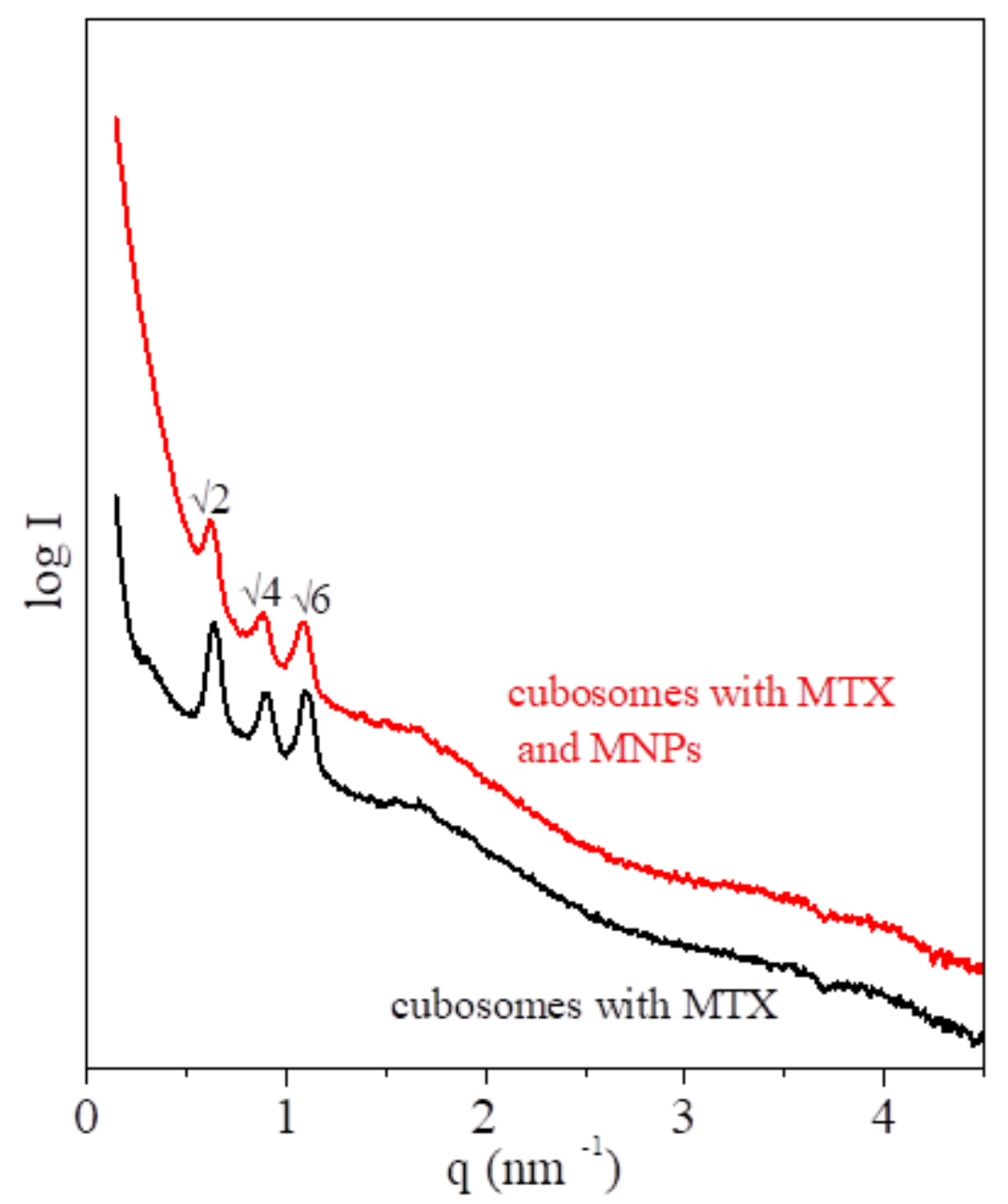

Fig. 6. SAXS spectra of the cubosomes and magnetocubosomes with Methotrexate at room temperature $\left(25^{\circ} \mathrm{C}\right)$.

Cryo-TEM allows imaging of the cubosomes without any changes of their structure. The CryoTEM images for cubosomes/magnetocubosomes with MTX shows a well-ordered structure inside the particles (Fig. S7). This highly ordered internal structure of the magnetocubosome provides an extensive hydrophobic-hydrophilic interfacial area, e.g., for the solubilization of different drugs and offers a convenient way for the delivery of the drug into the body. Additionally, the MNPs were visible inside the magnetocubosomes; hence magnetocubosomes were expected to be sensitive towards magnetic fields.

Low-Frequency Alternating Magnetic Field (AMF)-Stimulated Drug Release. 
The effect magnetic nanoparticles have on drug release, as well as other molecules from various lipidic structures, under the influence of the external magnetic field has been well documented. Vallooran et al. [27] showed that under a constant magnetic stimuli, the hexagonal phase with MNPs, aligns the orientation of the hexagonal domains in the direction of the external (constant) magnetic field, facilitating the transport of a hydrophilic drug across the liquid crystalline phase in this direction. Two cases were considered: when the magnetically induced orientation of the hexagonal domains in the membrane was in the direction of diffusion, effects the rate of transport of a hydrophilic drug, and when the forced position of the hexagonal domains was perpendicular to the diffusion direction, affects inhibition of drug diffusion. Mendozza et al. showed that the monoolein cubic phase with MNPs subjected to AMF for 10 minutes indicate Pn3m to $\mathrm{H}_{\text {II }}$ phase transition. [40]

Other works [28, 30, 32, 41, 42] rely on the so-called magnetothermal effect observed in an alternating magnetic field. This effect reveals itself as temperature rises, transferring heat to the immediate vicinity when the magnetic nanoparticles are exposed to the alternating magnetic field of sufficiently high frequency (typically from several $\mathrm{kHz}$ to hundreds of $\mathrm{kHz}$ ) due to the Néel and Brownian relaxation [43]. This heat transfer affects the physicochemical properties of the lipidic structures leading to a stimuli-response triggering drug release from lipid mesophases $[27,30]$. Since the heating efficiency, a prerequisite for the magnetothermal effect, varies as $\omega^{2} \tau /\left(1+\omega^{2} \tau^{2}\right)$, where $\omega$ is the magnetic field frequency and $\tau$ is the effective relaxation time, under LF-AMF conditions used in this work $(50 \mathrm{~Hz}$ AMF, $10 \mathrm{mT}, 20 \mathrm{~V})$ the relaxation time is only ca. $10^{-6} \mathrm{~s}$ [44], therefore the magnetothermal effect as the main stimulus for drug release has to be excluded. Other possible mechanism of triggered drug release includes changes of nanomechanical properties of the hybrid MNP - lipidic nanostructures. The incorporation of oleic acid-coated nanoparticles into the solid-supported BLMs as well as into the free-standing BLMs resulted in an increase of Young's modulus of elasticity measured by the nanoidentation and electrostriction methods [29]. This modulus is a measure of stiffness of lipid bilayer and an increase of this modulus renders the membrane prone to the mechanical rupture in an alternating magnetic field by the vibrating superparamagnetic nanoparticles localized inside the bilayer walls. Even though our hudrophobic nanoparticles (ca. $7 \mathrm{~nm}$ in diameter) seem to be too large to be embedded in the lipid bilayer of ca. 3-4 nm thick, there exist substantial literature proof of such structures $[42,43]$ The rupture of magnetoliposomes was confirmed by TEM and the release was confirmed be the doxorubicin outflow from magnetoliposomes loaded with this cytostatic drug and placed in the LF-AMF of $50 \mathrm{~Hz}$ frequency and $10 \mathrm{mT}$ magnetic field 
intensity [29]. Therefore, we used this approach to induce the methotrexate release from magnetocubosomes, assuming that mechanical vibration of magnetic nanoparticles rather than the localized heating of MNPs vicinity should lead to local disruption of magnetocubosomes and MTX release under LF-AMF.

A freshly prepared sample of $0.5 \mathrm{mg} / \mathrm{mL}$ magnetocubosome dispersion was placed inside the inductor coil. After a specified time, the absorbance was measured at $303 \mathrm{~nm}$ (Fig. 7). The application of AMF leads to an increase in the rate of drug release from the cubosomes. The relatively large data scatter, regardless of the number of repetitions is ascribed to the ex situ approach, where the sample is transferred from the inductor coil to the spectrophotometer chamber. Nevertheless, two important facts can be extrapolated from our results. First, the release takes place very fast and reaches saturation within the first $5 \mathrm{~min}$ of the application of AMF. This release appears to be much faster than in the case of magnetoliposomes [29]. After this time, the amount of drug released from the magnetocubosomes reaches $168 \mu \mathrm{g} / \mathrm{mL}$ (30\% of drug released compared to the initial drug load). Second, over the same time period, the release from magnetocubosomes without $\mathrm{AMF}$ stimulus was only ca. $10 \mu \mathrm{g} / \mathrm{mL}$, hence $2 \%$ of the drug released compared to the initial drug load. Due to the intense data scatter we could not assign the release results to any applicable release mechanism. The dashed curve shown in Fig. 7, even though obtained by fitting the results to the Korsmeyer-Peppas model for magnetocubosomes stimulated with AMF, is only a possible outcome. Nevertheless, it is clear that the application of LF-AMF triggers larger and faster release of the drug from magnetocubosomes. Furthermore, since the frequency of the AMF stimulus and the relaxation time of the magnetic nanoparticles used is insufficient to trigger the magnetothermal effect (vide supra and ref. 29), we presume that the alternating magnetic field at low frequency of $50 \mathrm{~Hz}$ caused mechanical vibration of the magnetic nanoparticles that can rupture to some extent the internal structure of magnetocubosomes facilitating the burst release of the drug from the aqueous channels of the cubosomes. We also admit that these disturbances can lead to destruction of the internal structure and possible phase transition based on [40]. 


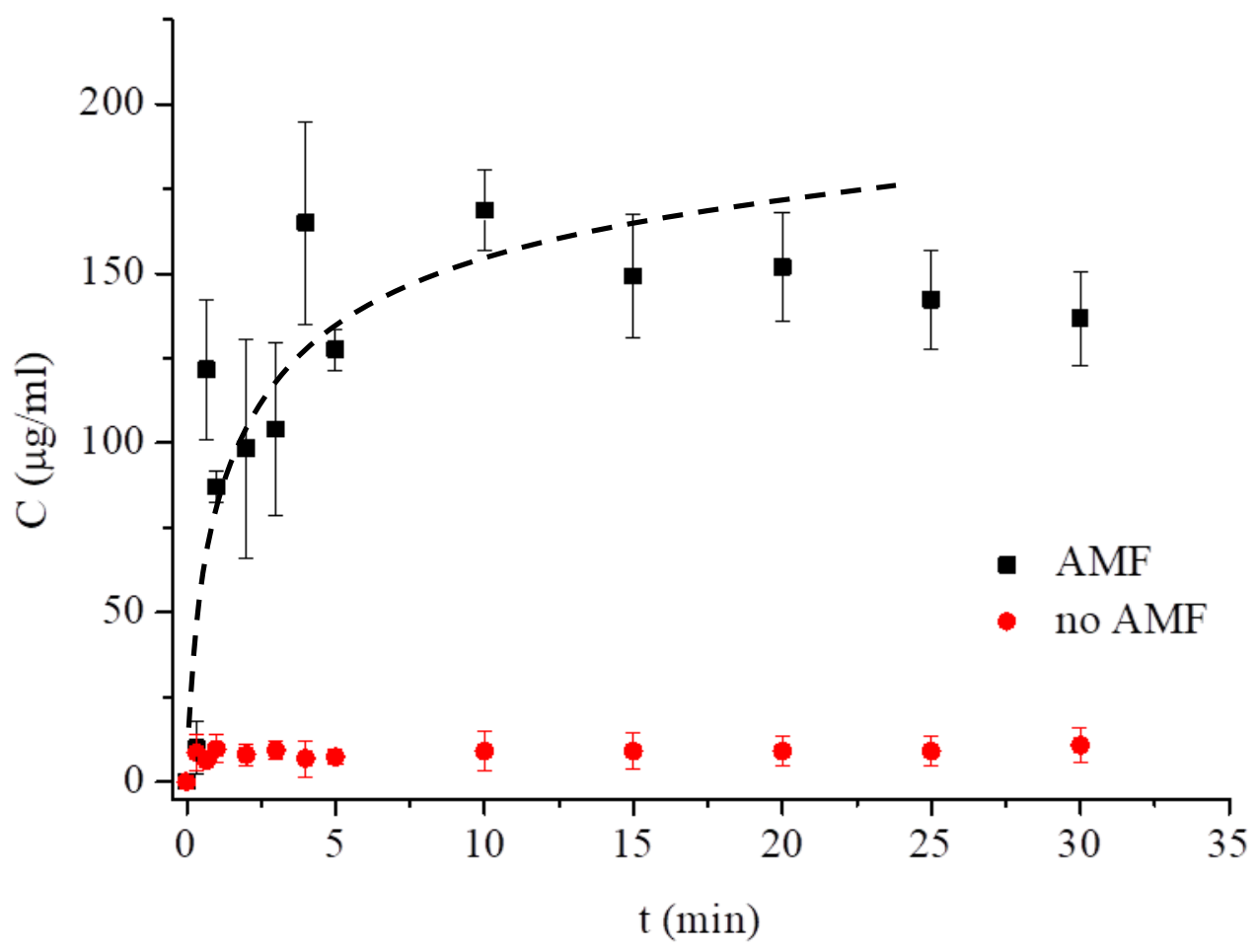

Fig. 7. MTX released from magnetocubosomes vs. time spontaneously and caused by LF-AMF at $\mathrm{pH} 7.4$ and $25^{\circ} \mathrm{C}$.

\section{Conclusions}

Methotrexate is a widely used drug; however, due to its toxic side-effects, it should be delivered to organisms encapsulated using an appropriate drug delivery vehicle preventing healthy cells from being exposed to its toxic influence. We have shown that a lipidic liquid crystalline cubic phase is a suitable matrix for such a task, decreasing its negative side effects. The release profiles of the drug from the cubic phases film are described by the Higuchi model. The removal of the drug is facilitated by an increase in temperature. Moreover, hydrophobic magnetic nanoparticles can be easily incorporated into the cubic phase nanoparticles. When low frequency alternating magnetic field is applied the release of MTX from the drug delivery vehicle is facilitated. These results show that magnetocubosomes are interesting biocompatible nanostructures providing high load of methotrexate compared to other carriers and giving the possibility of controlled rate and efficiency of methotrexate release at the appropriate sites by applying alternating magnetic field. This is promising and, therefore, requires further in vitro cytotoxicity and in vivo biodistribution investigations. 


\section{Acknowledgments}

This project was financed by the National Science Centre, conferred on the basis of decision number DEC-2016/23/B/ST4/03295. MM thanks Sinergia SNSF project no. CRSII2_154451 for support. CIISB research infrastructure project LM2015043 funded by MEYS CR is gratefully acknowledged for their financial support of the measurements at the CF Cryoelectron Microscopy and Tomography CEITEC MU. The authors would like to thank Dr. Jiri Novacek from CIISB for Cryo-TEM measurements and Aleksandra Rękorajska, University of Warsaw, for the synthesis of the magnetic nanoparticles. The authors would like to thank Dr. hab. Damian Pociecha, University of Warsaw, for the SAXS measurements of cubosomes.

\section{References}

1. Genestier, L.; Paillot, R.; Quemeneur, L.; Izeradjene, K.; Revillard, J.-P. Mechanisms of action of methotrexate. Immunopharmacology 2000, 47, 247-257, doi.org/10.1016/S0162-3109(00)00189-2

2. Bleyer, W. A. The clinical pharmacology of Methotrexate. Cancer 1978, 41, 36-51

3. Yoon, S.-A.; Choi, J. R.; Kim, J.-O.; Shin, J.-Y.; Zhang, X. H.; Kang, J.-H. Influence of Reduced Folate Carrier and Dihydrofolate Reductase Genes on MethotrexateInduced Cytotoxicity, Cancer Research and Treatment Search 2010, 42: p. 163-171, doi: $10.4143 /$ crt.2010.42.3.163

4. Kanarek, N.; Keys, H. R.; Cantor, J. R.; Lewis, C. A.; Chan, S. H.; Kunchok, T.; AbuRemaileh, M.; Freinkman, E.; Schweitzer, L. D.; Sabatini, D. M. Histidine catabolism is a major determinant of methotrexate sensitivity. Nature 2018, 559, $632-655$, doi.org/10.1038/s41586-018-0316-7

5. Rozensza, L. A.; Radnay, J. The effect of methotrexate on transformation and mitosis of normal human-blood lymphocytes in-vitro. Blood 1974, 43, 401 - 409,

6. Bookbinder, S. A.; Espinoza, L. R.; Fenske, N. A.; Germain, B. F.; Vasey, F. B. Methotrexate therapy in the rheumatic disease. Clinical and Experimental Rheumatology 1984, 2, 185 - 193,

7. Zeb, A.; Qureshi, O. S.; Kim, H.-S.; Cha, J.-H.; Kim, H.-S.; Kim, J.-K.; Improved skin permeation of methotrexate via nanosized ultradeformable liposomes. International Journal of Nanomedicine 2016, 11, 3813-3824, doi.org/10.2147/IJN.S109565 
8. Shabnam; Srinivas, P.; Ravindra Babu, D. S. Formulation and evaluation of parenteral methotrexate nanoliposomes. International Journal of Pharmacy and Pharmaceutical Sciences 2014, 6, 295-300,

9. Rosenholm, J. M.; Peuhu, E.; Bate-Eya, L. T.; Eriksson, J. E.; Sahlgren, C.; Linden, M.Cancer-cell-specific induction of apoptosis using mesoporous silica nanoparticles as drug-delivery vectors. Small 2010, 6, 1234-1241, doi: 10.1002/smll.200902355

10. de Oliveira Freitas, L. B.; Gonzalez Bravo, I. J.; de Almeida Macedo, W. A.; de Sousa, E. M. B. Mesoporous silica materials functionalized with folic acid: preparation, characterization and release profile study with methotrexate. Journal of Sol-Gel Science and Technology 2016, 77, 186-204, doi: 10.1007/s10971-015-3844-8

11. Kakkar, D.; Dumoga, S.; Kumar, R.; Chuttani, K.; Mishra, A. K.; PEGylated solid lipid nanoparticles: design, methotrexate loading and biological evaluation in animal models. Medicinal Chemical Communications 2015, 6, 1452-1463, doi:10.1039/C5MD00104H

12. Ferreira, M.; Silva, E.; Barreiros, L.; Segundo, M. A.; Costa Lima, S. A.; Reis, S. Methotrexate loaded lipid nanoparticles for topical management of skin-related diseases: Design, characterization and skin permeation potential. International Journal of Pharmaceutics 2016, 512, 14-21, doi.org/10.1016/j.ijpharm.2016.08.008

13. Hashada, R. A.; Ishaka, R. A. H.; Geneidia, A. S.; Mansoura, S. Methotrexate loading in chitosan nanoparticles at a novel pH: Response surface modeling, optimization and characterization. International Journal of Biological Macromolecules 2016, 91, 630639, doi.org/10.1016/j.ijbiomac.2016.06.014

14. Pretti, T. S.; Souza, M. A.; Santos, H. T.; Nascimento, A. C. G. G.; Santos, F. J.; Fraga, A. F.; Jafelicci, M. J.; Marques, R. F. C. Drug delivery nanotechnology applied to Methotrexate controlled release in human osteosarcona: in vitro test. Congreso Latino Americano de Orgaos Artificiais e Biomateriais 2012, http://www.metallum.com.br/7colaob/resumos/trabalhos_completos/08-020.docx

15. Guo, F.; Fan, Z.; Yang, J.; Li, Y.; Wang, Y.; Zhao, H.; Xie, L.; Hou, Z. A Comparative Evaluation of Hydroxycamptothecin Drug Nanorods With and Without Methotrexate Prodrug Functionalization for Drug Delivery. Nanoscale Research Letters 2016, 11, 384 - 396, doi: 10.1186/s11671-016-1599-y

16. Pereira, A. D. F.; Pereira, L. G. R.; Barbosa, L. A. D. O.; Fialho, S. L.; Pereira, B. G.; Patricio, P. S. D. O.; Pinto, F. C. H.; Da Silva, G. R. Efficacy of methotrexate-loaded 
poly(e-caprolactone) implants in Ehrlich solid tumor-bearing mice. Drug Delivery 2013, 2, 168-179, doi: 10.3109/10717544.2013.801052

17. Ghorbani, M.; Hamishehkar, H.; Arsalani, N.; Entezami, A. A. A novel dual-responsive core-crosslinked magnetic-gold nanogel for triggered drug release. Materials Science and Engineering C 2016, 68, 436-444, doi.org/10.1016/j.msec.2016.06.007

18. Lina, L.; Xua, W.; Lianga, H.; Hea, L.; Liua, S.; Lia, Y.; Lia, B.; Chena, Y.; Construction of $\mathrm{pH}$-sensitive lysozyme/pectin nanogel for tumor methotrexate delivery. Colloids and Surfaces B: Biointerfaces 2015, 126, 459-466, doi.org/10.1016/j.colsurfb.2014.12.051

19. Kulkarni, Ch. V.; Wachter, W.; Iglesias-Salto, G.; Engelskirchenb, S.; Ahualliac, S.Monoolein: a magic lipid? Physical Chemistry Chemical Physics 2011, 13, 30043021, doi: 10.1039/c0cp01539c

20. Nazaruk, E.; Szlęzak, M.; Górecka, E.; Bilewicz, R.; Osornio, Y. M.; Uebelhart, P.; Landau, E. M. Design and Assembly of pH-Sensitive Lipidic Cubic Phase Matrices for Drug Release. Langmuir 2014, 30, 1383-1390, doi.org/10.1021/la403694e

21. Negrini, R.; Sanchez-Ferrer, A.; Mezzenga, R.Influence of Electrostatic Interactions on the Release of Charged Molecules from Lipid Cubic Phases. Langmuir 2014, 30, 4280 - 4288, doi.org/10.1021/1a5008439|

22. Negrini, R.; Fong, W.-K.; Boyd, B. J.; Mezzenga, R. pH-responsive lyotropic liquid crystals and their potential therapeutic role in cancer treatment. Chemical Communications 2015, 51, 6671 - 6674, doi: 10.1039/c4cc10274f

23. Negrini, R.; Mezzenga, R. pH-Responsive Lyotropic Liquid Crystals for Controlled Drug Delivery. Langmuir 2011, 27, 5296-5303, doi: 10.1021/la200591u

24. Szlezak, M.; Nieciecka, D.; Joniec, A.; Pękała, M.; Gorecka, E.; Emo, M.; Stébé, M. J.; Krysiński, P.; Bilewicz, R. Monoolein cubic phase gels and cubosomes doped with magnetic nanoparticles - hybrid materials for controlled drug release. ACS Applied Materials and Interfaces 2017, 9, 2796-2805, doi: 10.1021/acsami.6b12889

25. Fong, W.-K.; Negrini, R.; Vallooran, J. J.; Mezzenga, R.; Boyd, B. J. Responsive selfassembled nanostructured lipid systems for drug delivery and diagnostics, Journal of Colloid and Interface Science 2016, 484: p. 320-339, doi.org/10.1016/j.jcis.2016.08.077 
26. Bonini, M.; Berti, D.; Baglioni, P. Nanostructures for magnetically triggered release of drugs and biomolecules. Current Opinion in Colloid and Interface Science 2013, 18, 459-467, doi.org/10.1016/j.cocis.2013.07.007

27. Vallooran, J. J.; Negrini, R.; Mezzenga, R. Controlling anisotropic drug diffusion in lipid- $\mathrm{Fe}_{3} \mathrm{O}_{4}$ nanoparticle hybrid mesophases by magnetic alignment. Langmuir 2013, 29, 999-1004, doi: 10.1021/la304563r

28. Monnier, C. A.; Burnand, D.; Rothen-Rutishauser, B.; Lattuada, M.; Petri-Fink, A. Magnetoliposomes: opportunities and challenges. European Journal of Nanomedicine. 2014, 6, 201-215, doi:10.1515/ejnm-2014-0042

29. Joniec, A.; Sek, S.; Krysinski, P. Magnetoliposomes as Potential Carriers of Doxorubicin to Tumours. Chemistry - A European Journal 2016, 22, 17715-17724, DOI : 10.1002/chem.201602809

30. Montis, C.; Castroflorio, B.; Mendozza, M.; Salvatore, A.; Berti, D.; Baglioni, P. Magnetocubosomes for the delivery and controlled release of therapeutics. Journal of Colloid Interface Science 2015, 449, 317-326, doi.org/10.1016/j.jcis.2014.11.056

31. Hong, S.K.; Kim, J.C. Cubic phase magnetic nanoparticles. Molecular Crystals and Liquid Crystals 2015, 607, 123-134, doi.org/10.1080/15421406.2014.939602

32. Wang, M.H.; Kim, J.-C. Magnetic field-responsive cubosomes containing magnetite and poly(N-isopropylacrylamide). Journal of Controlled Release 2013, 172 e139, doi.org/10.1016/j.jconrel.2013.08.225

33. Costa, P.; Sousa Lobo, J. M. Modeling and comparison of dissolution profiles. European Journal of Pharmaceutical Sciences 2001, 13, 123-133, doi.org/10.1016/S09280987(01)00095-1

34. Hyde, S. T.; Andersson, S.; Ericsson, B.; Larsson, K. A Cubic Structure Consisting of a Lipid Bilayer Forming an Infinite Periodic Minimum Surface of the Gyroid Type in the Glycerolmonooleat-Water System. Zeitschrift fur Kristallographie 1984,168, 213 219 ,

35. Briggs, J.; Chung, H.; Caffrey, M. The Temperature-Composition Phase Diagram and Mesophase Structure Characterization of the Monoolein/Water System. Journal de Physique II 1996, 6, 723-751,

36. Qiu, H.; Caffrey, M. The Phase Diagram of the Monoolein/Water System: Metastability and Equilibrium Aspects. Biomaterials 2000, 21, 223-234, doi.org/10.1016/S01429612(99)00126-X 
37. Pontinha, A. D. R.; Jorge, S. M. A.; Diculescu, V. C.; Vivan, M.; Oliveira-Brett, A. M. Antineoplasic Drug Methotrexate Redox Mechanism Using a Glassy Carbon Electrode. Electroanalysis 2012, 24, 917-923, doi-1org10000b51947ee.han.buw.uw.edu.pl/10.1002/elan.201100558

38. Gao, L.; Wu, Y.; Liu, J.; Ye, B. Anodic voltammetric behaviors of methotrexate at a glassy carbon electrode and its determination in spiked human urine. Journal of Electroanalytical Chemistry 2007, 610, 131-136, doi.org/10.1016/j.jelechem.2007.07.030

39. Acharya, D. P.; Moffat, B. A.; Polyzos, A.; Waddington, L.; Coia, G.; Wright, D. K.; Wang, H. X.; Egan, G. F.; Muir, B. W.; Hartley, P. G. Cubic mesophase nanoparticles doped with superparamagnetic iron oxide nanoparticles: a new class of MRI contrast agent. RSC Advances, 2012, 2, 6655-6662, doi:10.1039/C2RA20135F

40. Mendozza, M.; Montis, C.; Caselli, L.; Wolf, M.; Baglionia, P.; Berti, D. On the thermotropic and magnetotropic phase behavior of lipid liquid crystals containing magnetic nanoparticles. Nanoscale, 2018, 10, 3480-3488, doi: 10.1039/c7nr08478a

41. Reimhult, E. Nanoparticle-triggered release from lipid membrane vesicles. New Biotechnology 2015, 32, 665 - 672, doi.org/10.1016/j.nbt.2014.12.002

42. Bixner, O.; Reimhult, E. Controlled megnetosomes: embedding of magnetic nanoparticles into membranes of monodisperse lipid vesicles. Journal of Colloid and Interface Science 2016, 466, 62 - 71, doi.org/10.1016/j.jcis.2015.11.071

43. Shaghasemi B.S.; Virk M.M.; Reimhult E. Optimization of Magneto-thermally Controlled Release Kinetics by Tuning of Magnetoliposome Composition and Structure. Scientific Reports 2017, 7: 7474, doi.org/10.1038/s41598-017-06980-9

44. Fortin, J.-P.; Gazeau, F.; Wilhelm, C. Intracellular heating of living cells through Néel relaxation of magnetic nanoparticles. European Biophysics Journal 2008, 37, 223-228, doi: 10.1007/s00249-007-0197-4 